\title{
LOS HUMEDALES EN MÉXICO: TENDENCIAS Y OPORTUNIDADES
}

\section{P. Moreno Casasola}

Departamento de Ecología Funcional, Instituto de Ecología A.C., Km 2.5 Ant carretera Coatepec no 35 I, Las Hayas, Xalapa, México. patricia.moreno@inecol.edu.mx

Los humedales han jugado un papel muy importante para la humanidad en todos los continentes y hoy en día su conservación ha cobrado importancia. Por citar solamente algunos ejemplos de su importancia en la historia de la humanidad, los valles fértiles del Nilo, Eúfrates y Tigris dieron lugar a las culturas de los egipcios y sumerios (HAMMERTON, 1972; BOULÉ, 1994). En las regiones americanas de grandes humedales como el Amazonas, la densidad de población fue de 10 a 20 veces mayor que en las tierras altas de los alrededores (DENEVAN, 1976). En México varias culturas guardaron una estrecha relación con los humedales. Los olmecas surgieron en las planicies costeras inundables del sur de Veracruz y Tabasco, en el centro del Golfo de México, zona intensamente irrigada por los numerosos afluentes de los ríos Papaloapan, Coatzacoalcos y Tonalá (COE \& DIEHL, 1980). Los aztecas se establecieron sobre un lago y una buena parte de su agricultura estuvo ligada al uso de humedales, por medio del cultivo en chinampas (ROJAS, 1990). Hay reportes de la presencia de canales en el sur de Quintana Roo y Río Candelaria y sus tributarios (SIEMENS \& PULESTON, 1972; POPE \& DAHLIN, 1989), pertenecientes a la cultura maya lo cual implica un manejo de los humedales.

Los humedales conforman una enorme variedad y riqueza de comunidades vegetales con distinta composición, formas de vida y estructura. Frecuentemente se les considera como un sólo tipo de ecosistema, comparable a los bosques o pastizales. Sin embargo, los humedales agrupan gran parte de la variabilidad ambiental que se puede encontrar entre los ecosistemas más secos y forman una serie de tipos que de manera general son comparables, difiriendo principalmente en su grado de humedad o inundación (WHEELER et al., 2002). Por tanto, la hidrología, es decir la cantidad, calidad y estacionalidad de la inundación es el principal factor ambiental que determina y afecta a los humedales, y cuando están cerca de la costa, la salinidad es otro factor que produce heterogeneidad ambiental. Ello tiene como resultado humedales herbáceos y arbóreos, temporales y permanentes, de agua dulce, salinos e hipersalinos.

Los humedales de México ocupan una extensión mayor a lo largo de la costa que tierra adentro (OLMSTED, 1993). Incluyen desde las lagunas costeras someras con sus pastizales marinos, marismas y oasis en los desiertos, manglares y petenes, humedales herbáceos de agua dulce (popales, tulares), palmares y selvas inundables. Esta gran variabilidad conjunta una enorme cantidad de especies de flora y fauna y por tanto una alta biodiversidad, a pesar de que algunos de ellos por si mismos sean poco diversos, como los manglares. 


\section{EL FUNCIONAMIENTO DE ESTOS ECOSISTEMAS Y LAS ALTERACIONES PRODUCIDAS}

La hidrología es el principal factor que determina y afecta a los humedales (MITSCH \& GOSSELINK, 2000; JUNK 2002). Muchas de las diferencias entre los distintos humedales se basan en características del hidroperiodo. Este es el patrón estacional del nivel del agua en un humedal y se define por su duración (tiempo que permanece la inundación), frecuencia (el número de veces que se inunda en un tiempo dado), profundidad y época de inundación. Afecta de manera importante la composición de especies, la estructura del suelo, los procesos metabólicos y frecuentemente abre el ecosistema a entradas y salidas laterales de materiales (BRINSON, LUGO \& BROWN, 1981).

En las últimas decenas de años, se han producido cambios importantes en la hidrología de las zonas tropicales. Los cambios hidrológicos se han producido por causas naturales y también como consecuencia de las actividades del hombre. Los humedales están íntimamente ligados a las cuencas de las que reciben escurrimientos de agua, sólidos suspendidos y nutrientes. Esto hace que los humedales sean muy vulnerables a un manejo inadecuado de la cuenca (JUNK, 2002) y ello frecuentemente afecta la hidrología. Entre las principales causas de degradación, en orden de importancia, están:

a) Desecamiento de humedales mediante canales que ayudan a desaguar o bien elevación del nivel del terreno cubriéndolos con tierra, fundamentalmente para actividades productivas, sobre todo ganadería. En las planicies costeras extensas del Golfo de México es frecuente ver potreros dominados por gramíneas tolerantes a la inundación y con flora remanente de los humedales.

b) Desecamiento para construcción de desarrollos inmobiliarios. Cabe decir que hoy en día, el uso de materiales como los geotextiles está permitiendo a las constructoras desarrollar complejos urbanos sobre humedales, como sucede en el sur del Puerto de Veracruz. c) Construcción de obras de infraestructura como caminos. Actúan como barreras que impiden y modifican los flujos hidrológicos y son el resultado de estudios de impacto ambiental mal desarrollados o evaluados.

d) Represamientos de agua. DE LA LANZA \& CACERES (2005) indica que el $17.61 \%$ del volumen de agua del país $\left(107,000\right.$ millones de $\left.\mathrm{m}^{3}\right)$, se localizan en las presas, es decir casi la cuarta parte. Este gran volumen de agua retenido ha alterado la hidrología de los humedales al modificar la cantidad de agua y la estacionalidad de la inundación en los humedales cuenca abajo. Así mismo modifican la cantidad de sedimentos que bajan hacia los humedales y hacia las playas.

e) Dragado de humedales y canalización para control de inundaciones y mosquitos.

f) Contaminación. El escurrimiento de contaminantes arrastrados por los ríos así como por flujos superficiales durante las lluvias, ha producido una fuerte contaminación (por plaguicidas, herbicidas y pesticidas utilizados en las actividades agropecuarias y aguas negras tanto domésticas como industriales) en los humedales, sobre todo en las zonas costeras.

g) Invasión de especies es otra causa importante que altera el funcionamiento de los humedales. Hay especies que han sido introducidas de manera accidental (y en ocasiones deliberada), y que están produciendo problemas importantes en los humedales como el lirio acuático (Eichhornia crassipes), la especie acuática sumergida Hydrilla verticillata, los pastos africanos forrajeros como Echinochloa pyramidalis, el mosquito de la malaria (Anopheles quadrimaculatus), la tilapia de Mozambique (Oreochromis mossambicus) (NIÑO \& LOT, 1983; NOVELO \& MARTÍNEZ, 1987; MCKAYE et al., 1995; JUNK, 2002; LÓPEZ-ROSAS et al., 2006). Especies nativas como el arbusto Mimosa pigra, también se han vuelto invasoras debido a la degradación de las condiciones de los humedales.

e) La extracción de agua subterránea para ciudades, industrias y actividades agropecuarias trae alteraciones hidrológicas en los humedales y modifica su funcionamiento (i.e. alrededores de la Ciudad de México). 


\section{TENDENCIAS DE CAMBIO Y FACTORES DETERMINANTES}

Los cambios en los humedales han sido documentados principalmente para los manglares. Para los humedales de agua dulce hay muy poca información, debido a la carencia de tipificaciones, inventarios y mapas. Ello dificulta la valoración de cuanto se ha perdido y su estado actual. Sin embargo, se pueden describir los principales factores que han producido cambios y las tendencias generales, permitiendo tener una idea de que tan grave es la pérdida y el deterioro y cuales son las principales tendencias.

\section{Demografia}

Como ya se mencionó, la mayoría de los humedales de México son costeros. En este sentido, los cambios demográficos y el desarrollo de la zona costera, están teniendo un impacto considerable en los humedales mexicanos y la tendencia es al aumento.

México ha tenido una alta tasa de crecimiento de población, lo cual ha producido presiones fuertes sobre los recursos naturales, tanto terrestres como acuáticos. En los últimos años los asentamientos poblacionales en las costas mexicanas han estado cambiando y en algunas regiones de manera muy importante. Esto tiene repercusiones importantes en la conservación y transformación de los humedales. En 1987, había 126 municipios costeros, y 20 años después hay 161. Ello habla de un incremento en número de pobladores y una densificación, además de un desarrollo económico en la zona costera. SAAVEDRA et al. (1999) indica que de acuerdo al censo de 1950 solo el 9\% de la población del país vivía en los municipios costeros, equivalente a 2.55 millones de habitantes. El censo del 2000 muestra un incremento al 13\% (15.1 millones). Actualmente, en las entidades costeras del país, reside el $54.05 \%$ de la población. Sin embargo, hay fuertes diferencias entre estados. Hay algunos en los cuales un alto porcentaje de la población vive en los municipios costeros (sobre todo aquellos ligados con el turismo y los servicios) y otros en que no. En la figura 1 puede verse el porcentaje de población para cada entidad federativa que vive en municipios costeros y el porcentaje que vive tierra adentro. Resaltan extremos como Jalisco, Yucatán, Michoacán, Oaxaca y Chiapas en franca diferencia con Sinaloa, Sonora, Campeche, Baja California, Baja California Sur y Quintana Roo.

En el período 1950-1970, la población del país creció a tasas elevadas, superiores al 3\% anual. En

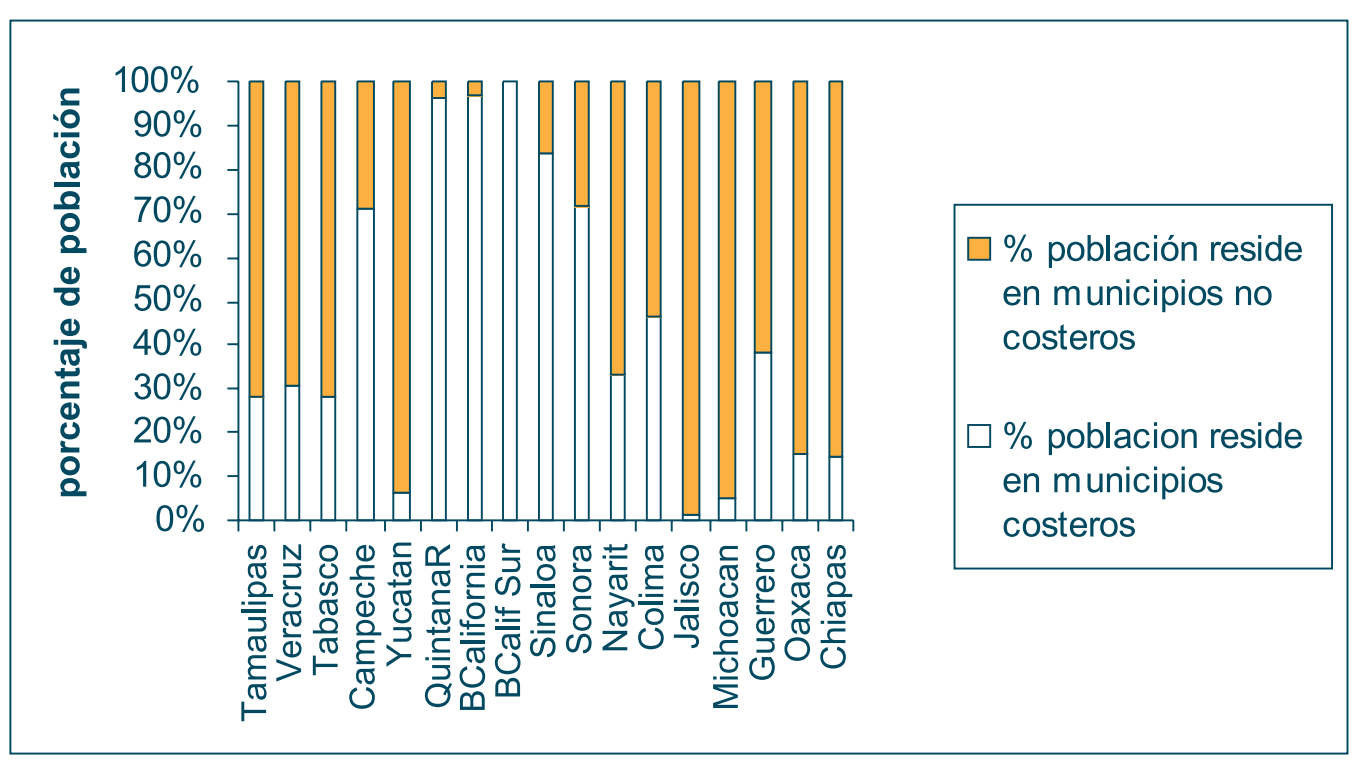

Figura 1. Porcentaje de la población estatal que vive en municipios costeros y porcentaje que vive en municipios al interior del estado (INEGI, 2001). Resaltan extremos como Jalisco, Yucatán, Michoacán, Oaxaca y Chiapas en franca diferencia con Sinaloa, Sonora, Campeche, Baja California, Baja California Sur y Quintana Roo. 
ese período los municipios costeros registraron tasas aún mayores, superiores al $4 \%$ anual, para posteriormente disminuir de manera significativa entre 1970 y 1980, aunque todavía crecían a ritmos mayores que los registrados a nivel nacional. De 1980 a 1995 la tasa de crecimiento a nivel nacional disminuyó (2.05\% para el periodo 1990-1995), presentándose este fenómeno también en los municipios costeros (2.71\% para el periodo 1990-1995). Sin embargo, en la costa las corrientes migratorias importantes alimentaron el crecimiento demográfico (SAAVEDRA et al., 1990). La migración se ha dado sobre todo en municipios costeros con fuerte actividad económica. Las distintas regiones costeras del país presentan tasas de crecimiento diferentes, siendo mayores para el Caribe, y menores para el Golfo de México (figura 2).

Con respecto a la densidad de población a nivel nacional, en el período de 1950 a 1995 casi se cuadruplicó, pasando de 13 habitantes por $\mathrm{km}^{2}$ a 46. Ya para 1950 los municipios costeros del Golfo tenían una densidad poblacional bastante más alta (18 hab $\left./ \mathrm{km}^{2}\right)$ que los del Caribe $\left(2 \mathrm{hab} / \mathrm{km}^{2}\right)$ y los del Pacífico (5 hab/ $\mathrm{km}^{2}$ ). Para 1995, la densidad poblacional del Caribe aumentó 7 veces, la del Pacífico poco más de 6 veces y la del Golfo casi se cuadruplicó (SAAVEDRA et al., 1990). Estos datos muestran que México, igual que una gran mayoría de los países del mundo, camina hacia una litoralización, es decir hacia una urbanización de la zona costera.

Este incremento de población en la zona costera constituye una amenaza para los humedales, ya que una buen parte del crecimiento urbano y agropecuario se dará sobre estos ecosistemas. Además, la pérdida pone en riesgo a la propia población costera, al perderse los servicios ambientales de protección a la línea de costa y de zona de acumulación y percolación de las grandes avenidas de agua producidas por tormentas y huracanes.

\section{Desarrollo económico}

México es un país en desarrollo que requiere diversificar sus actividades, generar empleos y elevar el nivel de vida de sus pobladores. Ello ha llevado a impulsar programas importantes para desarrollar económicamente la zona costera, los cuales incluyen desde la generación de energía, el desarrollo y crecimiento portuario, el establecimiento de nuevos enclaves turísticos y corredores como el de CancúnTulum.

Muchos políticos y una buena parte de la sociedad consideran que la protección ambiental

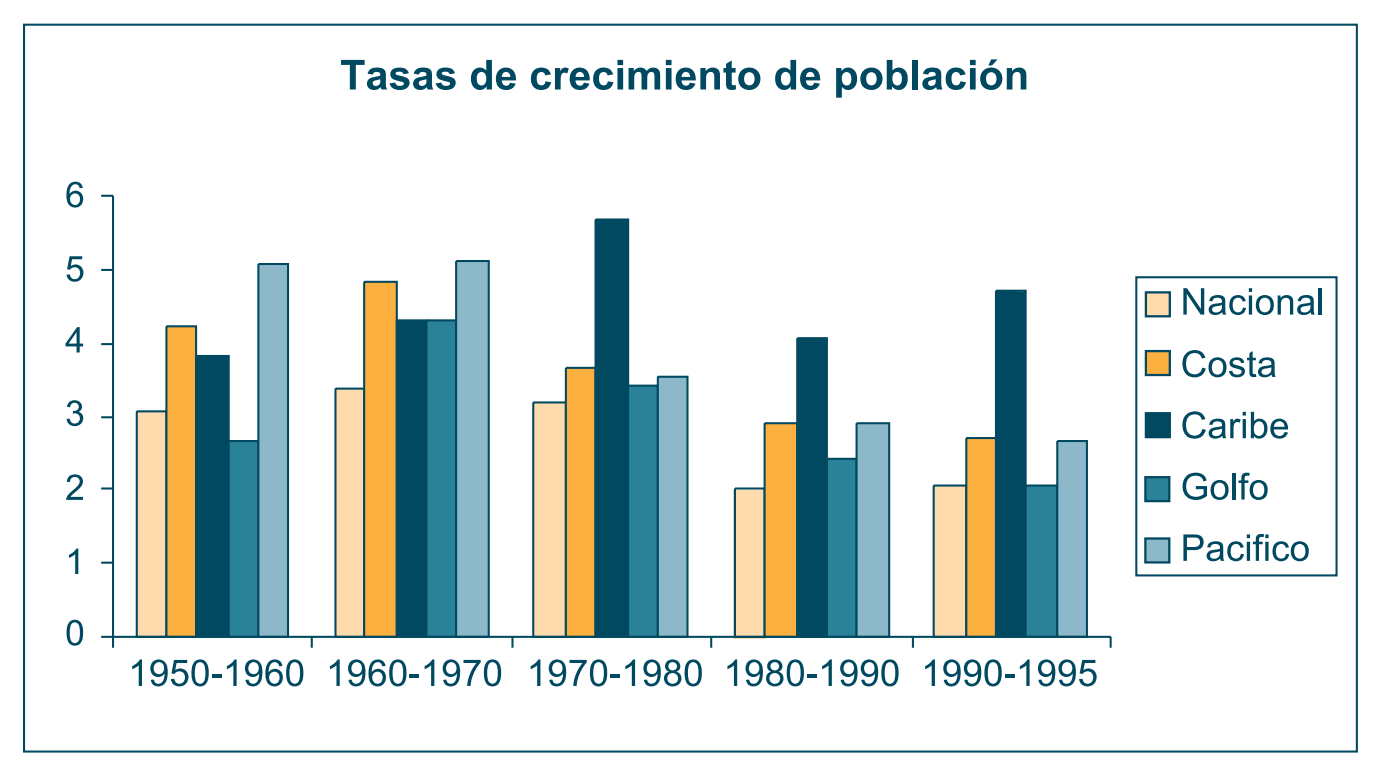

Figura 2. Tasas de crecimiento de la población a nivel nacional y en la zona costera de México durante el período 1950-1995 (datos de SAAVEDRA et al., 1999). 
impide el desarrollo. Sin embargo, se ha visto que la pobreza es en parte consecuencia de la degradación ambiental (BARBIER 2002; MILLENIUM ECOSYSTEM ASSESSMENT, 2005). Hoy en día, un desarrollo económico poderoso frecuentemente conlleva a una transformación importante de la naturaleza. Su economía y política relativamente estables son detonadores importantes del desarrollo económico, dando lugar a la existencia de grandes proyectos y de financiamiento para los mismos. La planificación e instrumentación de estos grandes proyectos, frecuentemente está basada en estudios de impacto ambiental inadecuados, con fuertes impactos negativos en el ambiente (LAURANCE et al., 2001) y México no es una excepción. Los impactos ambientales se ven como un mero trámite engorroso y no como una aportación a la mejora ambiental del proyecto. Los grandes proyectos de desarrollo han producido alteraciones directas e indirectas y esto puede verse de manera importante en los humedales costeros.

Desarrollo turistico, urbano e industrial. En regiones con fuerte presión para desarrollar proyectos turísticos como el litoral de Quintana Roo y zonas más puntuales como Bahías de Huatulco y Puerto Vallarta, muchos de los humedales han desaparecido y han sido sustituidos por las construcciones urbanas y de infraestructura. El crecimiento de la propia industria y de los desarrollos turísticos y portuarios ha traído consigo el crecimiento de zonas urbanas i.e. la expansión del Puerto de Veracruz sobre médanos y humedales. Esto se ha dado tanto de manera legal, como mediante asentamientos ilegales que van rellenando los humedales.

Energía (hidrocarburos, energía hidroeléctrica y nuclear). La cuenca del Gran Caribe es una importante zona de producción de petróleo. En 1994 produjo el 15\% a nivel mundial, siendo los principales Estados Unidos, Venezuela y México. Para México, el petróleo es el recurso más valioso de la zona costera actualmente y el que mayores divisas le produce. Ocupa el octavo lugar mundial en reservas de petróleo, calculadas para 51 años (INEGI-SEMARNAP, 1998). Desde la década de los 80 s hasta la fecha, más del $90 \%$ de la producción de petróleo y gas proviene de los estados costeros, en campos petroleros ya sea en la planicie costera o en la plataforma continental (PEMEX, 2003) del Golfo de México. Ello ha traído la construcción de infraestructura carretera, plantas industriales y desarrollos urbanos para dar hogar a los trabajadores que llegan, y mucho de ello se da sobre las extensas planicies de inundación como son las del sur de Veracruz y Tabasco. Otro de los fuertes impactos ambientales de la infraestructura de PEMEX son los viaductos, los cuales atraviesan extensas superficies, algunas con ecosistemas frágiles como los humedales en los que modifican el flujo de agua y alteran su funcionamiento. Hay más de 12,000 kilómetros de viaductos cruzando por el país. No hay una evaluación de los cambios que esto ha traído en el funcionamiento de los humedales, y las distintas Normas Oficiales Mexicanas de PEMEX no contemplan la mitigación de los impactos ambientales a estos ecosistemas.

La generación de electricidad cuenta con extensas obras en la zona costera del Golfo de México, incluyendo la única planta nucleoléctricas con que cuenta el país. La principal consecuencia se produce por el uso de agua para enfriamiento, la cual se obtiene de humedales y posteriormente sale al ambiente a una temperatura más elevada.

\section{Actividades agropecuarias y de acui- cultura}

La transformación más generalizada se ha debido al crecimiento de la frontera agropecuaria. Se ha hecho de manera lenta pero con carácter extensivo. Ello ha traído la tala y quema de la vegetación, el drenaje y relleno de humedales con la finalidad de convertirlos en "campos aptos para la actividad productiva". Las consecuencias han sido modificaciones en la composición de especies, pérdida de biodiversidad y alteraciones en el funcionamiento. Frecuentemente implican obras de drenaje de humedales alterando de manera permanente la hidrología. El bajo precio de la leche y la carne para los productores y las prácticas de manejo extensivo del ganado hacen que haya una presión constante sobre los humedales para incorporarlos a los terrenos productivos ganaderos. 


\section{Contaminación}

La tendencia hacia la urbanización está produciendo una mayor generación de aguas contaminadas en las poblaciones, las cuales frecuentemente son colectadas en los sistemas de drenaje y vertidas en uno o pocos puntos. En algunas ocasiones hay plantas de tratamiento para recibirlas, pero en la mayoría de los casos son vertidas a cuerpos de agua y a humedales. Se ha rebasado la capacidad de estos sistemas para limpiar el agua, servicio que prestaron a la humanidad durante cientos de años. En los municipios una parte considerable de los fondos está destinado para la construcción de infraestructura que incluye las plantas de tratamiento (generalmente de tratamiento primario únicamente), pero son contados los casos en que éstas funcionan adecuadamente. Por tanto, en los próximos años hay una tendencia a incrementar los niveles de contaminación en humedales, cuerpos de agua y aún manto freático.

El enriquecimiento de las aguas por los nutrientes arrastrados produce cambios en la composición y estructura de las comunidades. La eutrofización conlleva un cambio en las poblaciones de algas y la explosión de las poblaciones de algunas fanerógamas también se ha visto relacionada con esta mayor disponibilidad de nutrientes (i.e. Pistia stratiotes, Typha domingensis, Eichhornea crassipes).

\section{Mayor vulnerabilidad all cambio climático}

El deterioro de los humedales por las causas arriba expuestas, los hace más vulnerables a otro tipo de impactos como lo que conlleva el cambio climático. Tanto las sequías como el incremento en la cantidad y temporalidad del agua son afectaciones a la hidrología del humedal, que producen alteraciones en su funcionamiento. Un humedal degradado tiene menor capacidad de respuesta a modificaciones en su hidrología. Ello tiene como consecuencia una mayor vulnerabilidad de las poblaciones costeras a impactos como tormentas y huracanes. La modificación de la hidrología ha hecho que los humedales pierdan su capacidad para funcionar como esponjas que retengan y liberen el exceso de agua.

\section{EL FUTURO. OPORTUNIDADES}

El uso de los bienes y servicios que proveen los humedales a la sociedad implica la conservación de la integridad ecológica de estos ecosistemas, definida como la capacidad para soportar o mantener un sistema biológico natural y equilibrado (KARR, 1996). Es necesario trabajar en opciones y estrategias para asegurar que bajo el panorama de crecimiento y desarrollo de la zona costera se conserva la biodiversidad de los humedales, su funcionamiento y la fuente de agua de la cual se alimentan. Existen alternativas, tanto contempladas en la propia legislación mexicana como fuera de ella, que es necesario reforzar.

\section{Desarrollo socioeconómico planifi- cado}

i. Ordenamiento del territorio. Este instrumento legal contemplado en la legislación ambiental mexicana puede generar una planificación a nivel municipal de uso del suelo que ponga límites a los cambios de uso del suelo para fines agropecuarios, que garantice que no se llevan a cabo desarrollos de infraestructura en humedales, que se establecen áreas de conservación y zonas de amortiguamiento alrededor de los principales humedales, e identifica los puntos de descargas de aguas contaminadas y las necesidades de establecimiento de plantas de tratamiento. El desarrollo económico y los proyectos de atracción de inversión y migración hacia la zona costera solamente deben llevarse a cabo con base un ordenamiento territorial, que además siente las bases para un manejo integral de la zona costera (MIZC). Una de las principales formas de mitigación para el impacto del cambio climático en los humedales es la planificación adecuada, mediante la creación de escenarios que permitan anteponer la seguridad de la población al desarrollo de infraestructura.

\section{ii. Alternativas para el desarrollo sustentable.}

El Plan Nacional de Desarrollo del país plantea como uno de sus objetivos el desarrollo sustentable. Igual que en el caso anterior, este es un paso importante. Sin embargo, las políticas actuales de medio ambiente no reflejan esta visión. Nuevamente se carece de una política sobre medio ambiente y sobre desarrollo sus- 
tentable, que asegure la conservación y uso sustentable de los humedales. Es necesario, sobre la base legal ya existente, trabajar en mecanismos y estrategias para instrumentar los programas de desarrollo sustentable como verdaderas alternativas económicas y darles el peso a los temas ambientales, incluyendo el ciclo del agua y los humedales. Así mismo, se debe fomentar el uso de biotecnología para conservar y restaurar los humedales (limpieza del agua, control de erosión, retención de bordes, etc.)

iii. La frontera agropecuaria y las prácticas productivas. Es necesario armonizar las prácticas productivas con la conservación y manejo sustentable de humedales. La introducción de especies exóticas debe hacerse bajo planes de seguimiento y evaluación justificados. Es necesario crear transversalidad en tópicos ambientales entre los ministerios de producción agropecuaria, forestal y pesquera y los temas ambientales y de desarrollo sustentable.

iv. Transversalidad en las instituciones de gobierno. Una gran debilidad es que tanto en las diversas secretarias de gobierno como dentro de los propios inversionistas, sigue prevaleciendo la idea de que el proyecto manda sobre el ambiente, y éste último tiene que adaptarse a las necesidades. Mientras no haya un reconocimiento y aceptación real de que la naturaleza y los ecosistemas imponen limites al desarrollo y al crecimiento y que los proyectos son los que deben adaptarse al ambiente, no podrá darse un desarrollo sustentable. Es necesario trabajar en mecanismos y estrategias de transversalidad en los temas ambientales, y ello constituye uno de los principales planteamientos del MIZC.

\section{Protección y conservación de humedales}

i. Reservas de la Biosfera y ANPs. El incremento en el número de áreas protegidas en los últimos 10 años ha sido muy importante. El país actualmente cuenta con 164 áreas naturales de carácter federal que comprenden 23,048,994 hectáreas, dentro de distintas categorías (www.conanp.gob; www.iberomab.org) y que incluyen 36 reservas de la biosfera. Además en varios estados existen áreas naturales estatales que también incluyen humedales. La categoría que más se ha consolidado es la de Reservas de la Biosfera, ya que la mayoría cuentan con planes de manejo, personal y presupuesto. Es necesario avanzar aún más en este sentido para las otras categorías estatales y federales.

Uno de los problemas a que se enfrentan estas ANPs es que frecuentemente dentro de su superficie no está contemplada la fuente de agua que mantiene a los humedales ni los diversos vínculos que mantienen con otros ecosistemas. Los ríos, escurrimientos o zonas de infiltración de agua que posteriormente aflora a los humedales quedan fuera del ANP. Ello hace vulnerables a estos ecosistemas y de alguna manera es necesario que los planes de manejo tomen en cuenta esta situación. Una segunda problemática es que no se ha llegado a consolidar totalmente el sistema de ANPs federales y menos aún los estatales. En varios de ellos aún se carece de personal pagado, planes de manejo y recursos anuales para garantizar un funcionamiento mínimo.

ii. Incorporación de nuevas figuras de conservación y manejo de humedales. México ha tenido un gran crecimiento en el número de sitios Ramsar. Hoy en día México cuenta con 112 humedales prioritarios Ramsar abarcando una superficie de alrededor de 8 millones de ha. Cabe decir que algo mas de la tercera parte de los sitios Ramsar también cuentan con otra categoría de protección como es Parque Nacional o Reserva de la Biosfera. La categoría de humedal prioritario Ramsar no implica la necesidad de compra o expropiación de tierras lo cual permite en un país con la población de México, la creación de áreas con un manejo sustentable que garantice su conservación. Uno de los compromisos de México para cada sitio Ramsar, es contar con un plan de manejo, el cual debe tener un fuerte componente participativo del gobierno y de la sociedad (www. ramsar.org). Ello proporciona una alternativa importante para desarrollar planes de manejo sustentables de humedales en todas las regiones del país.

\section{Generación de información}

i. Investigación básica. En este sentido, la falta de conocimiento que se tiene de estos ecosistemas en zonas tropicales, tanto a nivel científico como a nivel 
de la sociedad, hacen que sean uno de los ambientes más amenazados. A su vez, la integridad ecológica de los humedales depende de formas integradas de manejo de cuencas, lo cual implica numerosos usos, actores e intereses, no necesariamente relacionados de manera directa con los humedales. Esto requiere un importante nivel de conocimiento de las interacciones ecológicas y socioeconómicas, ausente en la mayor parte de los países (JUNK, 2002), incluyendo México. Es importante generar líneas de trabajo sobre diversos temas de humedales, desde la hidrología hasta la ecología y ecofisiología y ligarlos con esquemas de investigación multidisciplinarios con participación de otras áreas de conocimiento, i.e. antropología, sociología, etc.

\section{ii. Evaluación y valoración de los servicios} ambientales. Los humedales prestan numerosos servicios ambientales de gran valor a la sociedad (Martínez et al., 2007). Sin embargo la sociedad en general y los políticos lo desconocen y no lo toman en cuenta. Es necesario generar información local sobre estos servicios ambientales que integre las visiones de los pobladores y que ayude a conservar el ambiente que hoy en día les da trabajo, hogar y alimento.

\section{Gobernanza y solución de conflictos entre usuarios}

i. Creación de redes de usuarios. La participación social es fundamental en la conservación y manejo de los recursos y del medio ambiente. Los humedales generalmente son áreas de conflicto entre sectores con distintas visiones, i.e. desecación para ganadería y urbanización versus conservación del agua y pesca. Es importante crear foros de trabajo (por ej. para el desarrollo de los planes de manejo de sitios Ramsar) y que como segunda etapa constituyan redes de comunicación, relación e interacción, que le dé sostenibilidad a los procesos.

Finalmente, la gran cantidad de actores que tienen intereses en los humedales hace necesario contar con políticas nacionales de humedales claramente establecidas, mecanismos de interacción, trabajo conjunto y concertación así como estructuras para su aplicación (JUNK, 2002). Esta visión aún no ha logrado instrumentarse en México.

\section{BIBLIOGRAFÍA}

BARBIER, E.B. 2000. The economic linkages between rural poverty and land degradation: some evidence from Africa. Agriculture, Ecosvstems and Environment 82: 355-370.

BOULE, M.E. 1994. An early history of wetland ecology. En: W.J. Mitsch (ed). Global wetlands: Old World and New. Elsevier. Amsterdam: 5774.

BRINSON, M.M., LUGO, A.E. \& BROWN, S. 1981. Primary productivity, decomposition and consumer activity in freshwater wetlands. Annual Review of Ecology and Systematics 12: 123-161.

COE, M.D. \& DIEHL, R.A. 1980. In the land of the Olmec: the archaeology of San Lorenzo Tenochtitlan. University of Texas Press, Austin. Vol. I.

DE LA LANZA, G.E. \& CÁCERES, L.M. 1994. Lagunas costeras y el litoral mexicano. Universidad de Baja California Sur. México. 52pp.

DENEVAN, W.M. 1976. The aboriginal population of Amazonia. En: W.M. Denevan (ed.) The native population of the Americas. University of Wisconsin Press, Madison. 205-234.

HAMMERTON, D. 1972. The Nile river - a case study. En: R.T. Oglesby, C.A. Carlson y M.J. McCann (eds.). River ecology and man. Academic Press, Nueva York: 71-214.

INEGI. 2001. Estados Unidos Mexicanos. XII Censo general de población y vivienda, 2000. Tabulados Básicos. Aguascalientes, Ags. México.

INEGI-SEMARNAP. 1998 Estadisticas del medio ambiente. México, 1999. Volumen I y II. INEGI. Aguascalientes, México.

http://www.semarnat.gob.mx/estadisticas_ ambientales

JUNK, W.J. 2002. Long-term environmental trends and the future of tropical wetlands. Environmental Conservation 29 (4): 414-435.

LAURANCE, W.F., COCHRANE, M.A., BERGEN, S., FEARNSIDE, P.M., DELAMÔNICA, P., BARBER, C., D'ANGELO, S., FERNANDES, T. 2001. The future of the Brazilian Amazon. Science 291: 438-439.

LÓPEZ, H., MORENO-CASASOLA, P. \& MENDELSSOHN, I. 2006. Plant invasion of a tropical freshwater marsh: Response of the inva- 
ded community to experimental disturbances. Wetlands 26 (2): 593-604.

MARTÍNEZ, M.L., INTRALAWAN, A., VÁZQUEZ, G., PÉREZ-MAQUEO, O., SUTTON, P. \&. LANDGRAVE, R. 2007. The coasts of our world: Ecological, economic and social importance. Ecological Economics 63 (2-3): 254-272.

MCKAYE, K.R., RYAN, J.D., STAUFFER, JR., LÓPEZ PÉREZ, L.J., VEGA, G.I. \& VAN DEN BERGHE, E.P. 1995. African Tilapia in Lake Nicaragua: Ecosystem in transition. BioScience 45:406-411.

MILLENIUM ECOSYSTEM ASSESSMENT. 2005. Ecosystems and human well-being. Synthesis. Island Press, Wshington D.C.

MITSCH, W.J. \& GOSSELINK, J.G. 2000. Wetlands. John Wiley \& Sons. Nueva York. 3. Edición. 920 pp.

NIÑO, M. S. \& LOT, A. 1983. Estudio demográfico del lirio acuático Eichornia crassipes (Mart.) Solms: dinámica de crecimiento en dos localidades selectas de México. Boletín de la Sociedad Botánica de México 45: 71-83.

NOVELO, A. \& MARTÍNEZ, M. 1987. Hydrilla verticillata (Hydrocharitaceae), problemática maleza acuática de reciente introducción en México. Anales del Instituto de Biología UNAM. Serie Botánica, 58 (Núm. Único): 97-102.

OLMSTED, I. 1993. Wetlands of Mexico. En: D.F. Whigham, D. Dykyjová y S. Hejnÿ, (eds.)
Wetlands of the world I: inventory, ecology and management. Handbook of vegetation science. Kluwer Academic Publishers, Dordrcht: 637678.

PEMEX, 2003. http://www.pemex.com y http:// www.pemex.com/index.cfm/action/content/sectionID/1/catID/237/index.cfm

POPE, K. \& DAHLIN, B.H. 1989. Ancient Maya wetland agriculture: new insights from ecologi$\mathrm{cal}$ and remote sensing research. Journal of Field Archeology 16: 87-106.

ROJAS, R.T. 1990. La agricultura en la época prehispánica. En: R.T. Rojas. (coord.) La agricultura en tierras mexicanas desde sus orígenes hasta nuestros días. Consejo Nacional para la Cultura y las Artes- Grijalbo. México D.F.: 15-138.

SAAVEDRA, F., CIPRÉS, E. \& OSORIO, L. 1999. La situación sociodemográfica de las zonas costeras. En: R. Tuirán. (coord.). La situación sociodemográfica de México, 1999. Consejo Nacional de Población. México D.F. 73-90.

SIEMENS, A.H. \& PULESTON, D.E. 1972. Ridged fields and associated features in southern Campeche: new perspective on the lowlands Maya. American Antiquity 37: 228-239.

WHEELER, B.D., MONEY, R.P. \& SHAW, S.C. 2002. Freshwater wetlands. En: M:R: Perrow y A.J. Davy (Ed.). Handbook of ecological restoration. Vol 2. Restoration in practice. Cambridge University Press. Londres. 325-353. 